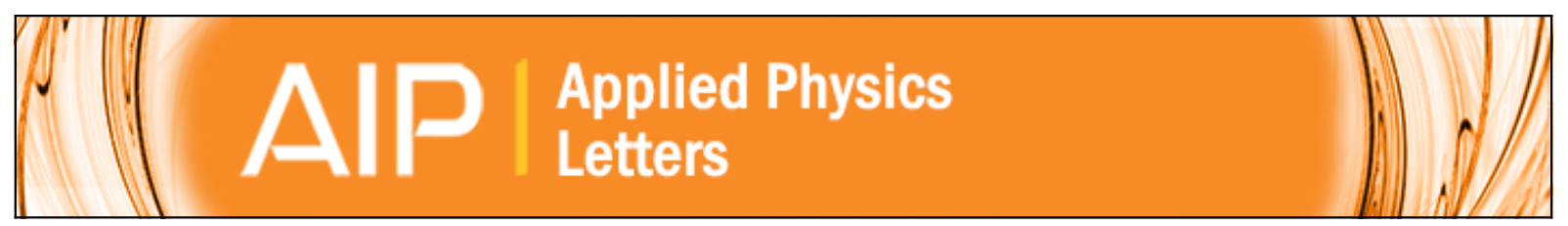

\title{
Work function of few layer graphene covered nickel thin films measured with Kelvin probe force microscopy
}

B. Eren, U. Gysin, L. Marot, Th. Glatzel, R. Steiner, and E. Meyer

Citation: Applied Physics Letters 108, 041602 (2016); doi: 10.1063/1.4940891

View online: http://dx.doi.org/10.1063/1.4940891

View Table of Contents: http://scitation.aip.org/content/aip/journal/apl/108/4?ver=pdfcov

Published by the AIP Publishing

\section{Articles you may be interested in}

Thin Ni film on graphene current spreading layer for $\mathrm{GaN}$-based blue and ultra-violet light-emitting diodes

Appl. Phys. Lett. 102, 151115 (2013); 10.1063/1.4802800

The interlayer screening effect of graphene sheets investigated by Kelvin probe force microscopy

Appl. Phys. Lett. 95, 222107 (2009); 10.1063/1.3269597

Measurements of metal gate effective work function by $\mathrm{x}$-ray photoelectron spectroscopy

J. Appl. Phys. 101, 074504 (2007); 10.1063/1.2713993

Atomic-layer-resolved local work functions of $\mathrm{Pb}$ thin films and their dependence on quantum well states

Appl. Phys. Lett. 90, 013109 (2007); 10.1063/1.2403926

A versatile Kelvin probe for dynamic work function change measurements during gas adsorption and in situ film growth experiments

Rev. Sci. Instrum. 68, 3866 (1997); 10.1063/1.1148039

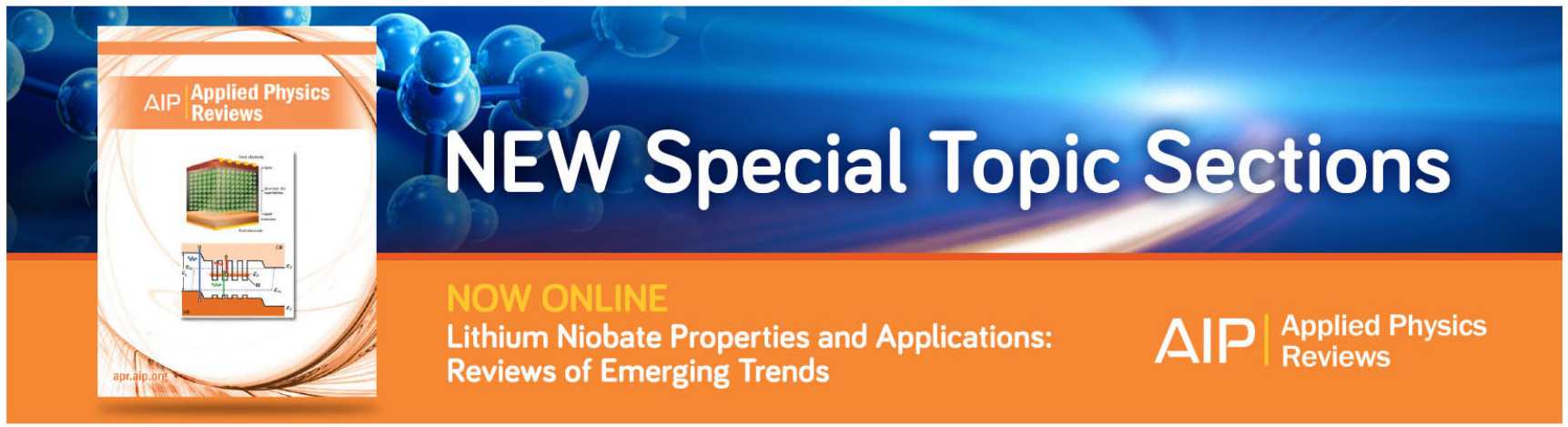




\title{
Work function of few layer graphene covered nickel thin films measured with Kelvin probe force microscopy
}

\author{
B. Eren, ${ }^{1,2}$ U. Gysin, ${ }^{1}$ L. Marot, ${ }^{1, a)}$ Th. Glatzel, ${ }^{1}$ R. Steiner, ${ }^{1}$ and E. Meyer ${ }^{1}$ \\ ${ }^{1}$ Department of Physics, University of Basel, Klingelbergstrasse 82, CH-4056 Basel, Switzerland \\ ${ }^{2}$ Material Sciences Division, Lawrence Berkeley National Laboratory, 1 Cyclotron Road, Berkeley, \\ California 94720, USA
}

(Received 13 August 2015; accepted 15 January 2016; published online 26 January 2016)

\begin{abstract}
Few layer graphene and graphite are simultaneously grown on a $\sim 100 \mathrm{~nm}$ thick polycrystalline nickel film. The work function of few layer graphene/Ni is found to be $4.15 \mathrm{eV}$ with a variation of $50 \mathrm{meV}$ by local measurements with Kelvin probe force microscopy. This value is lower than the work function of free standing graphene due to peculiar electronic structure resulting from metal 3d-carbon 2p( $\pi$ ) hybridization. @ 2016 AIP Publishing LLC. [http://dx.doi.org/10.1063/1.4940891]
\end{abstract}

Work function (WF) is one of the basic physical quantities related to the electronic structure and interactions at surfaces and interfaces. The graphene/Ni interface is an interesting system because the lattice match between the (111) facets of $\mathrm{Ni}$ and graphene results in a strong hybridization between metal $3 \mathrm{~d}$ and $\mathrm{C} 2 \mathrm{p}(\pi)$ states. ${ }^{1-7}$ So far, the WF of this system has been evaluated from electronic structure constructions with theoretical models, ${ }^{3,4}$ or with $\mathrm{x}$-ray absorption and x-ray emission spectroscopy, and electron energy loss spectroscopy measurements. ${ }^{5-7}$ The present study aims to measure the WF of this system directly with Kelvin probe force microscopy (KPFM) without the use of any structural models. Moreover, unlike spectroscopy techniques which average over a large area, KPFM can measure spatial distribution of WF over the surface.

Two most common chemical vapor deposition (CVD) processes for graphene growth on metals involve either surface precipitation of carbon from a supersaturated metal/carbon solution or hydrocarbon decomposition on a catalytic surface. ${ }^{1,2}$ Both pathways are available on Ni thin films since it has high carbon solubility and catalytically active sites due to surface corrugation. The former typically leads to few layer graphene (FLG) precipitation to the surface, whereas the latter leads to graphite layers taking the volume of metal during grain boundary motion. ${ }^{8}$ Two different growth mechanisms are explained in detail in Ref. 8. Here, we exploit the co-existence of graphite and FLG on the surface, and we perform KPFM to measure WF variations across such a surface. The WF of 3-4 layers of graphene on $\mathrm{Ni}$ is found to be $4.15 \mathrm{eV}$ with local variations of $\pm 50 \mathrm{meV}$.

The sample was prepared in a high-vacuum chamber with a base pressure better than $2 \times 10^{-8}$ mbar with the following steps: (1) magnetron sputtering of $100 \mathrm{~nm}$ AlN on $\mathrm{Si}(111)$ which acts against diffusion of Si into the metal and (2) magnetron sputtering of $100 \mathrm{~nm}$ thick $\mathrm{Ni}$ film at $0.02 \mathrm{mbar}$ Ar pressure with a coating rate of $20 \mathrm{~nm} / \mathrm{min}$. In both steps, water-cooled magnetrons were operated in a pulsed-DC mode with $496 \mathrm{~nm}$ pulse width at $50 \mathrm{kHz}$ frequency at a power of $50 \mathrm{~W}$ while the samples were kept at

\footnotetext{
${ }^{\text {a) }}$ Author to whom correspondence should be addressed. Electronic mail: Laurent.marot@unibas.ch
}

room temperature (RT). (3) Sample temperature was ramped up to $840{ }^{\circ} \mathrm{C}$ within $4 \mathrm{~min}$ in high-vacuum and kept for 5 more minutes in $0.06 \mathrm{mbar}$ acetylene $\left(\mathrm{C}_{2} \mathrm{H}_{2}\right)$. The temperature was dropped to $790^{\circ} \mathrm{C}$ when $\mathrm{C}_{2} \mathrm{H}_{2}$ was introduced. We should note that the choice of this low temperature is intentional because at $900^{\circ} \mathrm{C}$ and above, recrystallization of $\mathrm{Ni}$ is saturated; meaning that the grain boundary motion necessary for graphite growth will be negligibly small. ${ }^{8}$ Temperature measurements of the sample surface were done with a twowavelength pyrometer. Considering the thickness of the $\mathrm{Ni}$ film and the classical C solubility of $\mathrm{Ni}(0.09-0.1 \mathrm{wt} . \%)^{9,10}$ at $790^{\circ} \mathrm{C}, 1-2$ layers of graphene should grow on the sample surface. Since the segregation at the grain boundaries increases the solubility significantly, ${ }^{11}$ and the newer in situ results suggest FLG growth already happens before the cooldown, ${ }^{12}$ a higher coverage (3-4 layers) can be anticipated.

Chemical analysis with $\mathrm{x}$-ray photoelectron spectroscopy (XPS) was done before and after CVD growth without breaking the vacuum using a VG ESCALAB 210 analyzer and a monochromatic $\mathrm{Al} \mathrm{K} \alpha$ source. Survey XPS spectrum consisted of peaks registered to $\mathrm{Ni}, \mathrm{C}$ (after CVD growth), and $\mathrm{O}$ (as surface impurity, less than 2 at. \%). Fitting procedure of XPS peaks is discussed elsewhere. ${ }^{13}$ As shown in Fig. 1(a), Ni was found only in the metallic chemical state. CVD grown carbon was found in the graphitic phase (Fig. 1(b)). Various microscopy techniques including optical, scanning electron microscopy (SEM), and Raman microspectroscopy were employed after storage in air. The dappled pattern in the SEM micrograph in Fig. 1(c) (acquired with a Hitachi S- 4800 device at $5 \mathrm{keV}$ acceleration voltage) results from the difference in the amount of secondary electrons in the first few atomic layers of the surface which is less for $\mathrm{C}$ than for $\mathrm{Ni},{ }^{14,15}$ meaning that the darker areas are graphite and brighter areas are FLG covered Ni. Using the atomic concentrations from the XPS analysis (22 at. \% Ni and 78 at. \% C) and assuming graphite attenuates all photoelectrons from underlying $\mathrm{Ni}$, the solution of the thickogram equation yields $1.1 \mathrm{~nm}$ of carbon, ${ }^{16}$ equivalent to 3-4 layers of graphene covering the bright areas in Fig. 1(c).

Raman spectroscopy measurements were performed with a WITec alpha 300 confocal Raman microscope, equipped with a $532 \mathrm{~nm}$ wavelength laser with $2.1 \mathrm{~mW}$ laser 


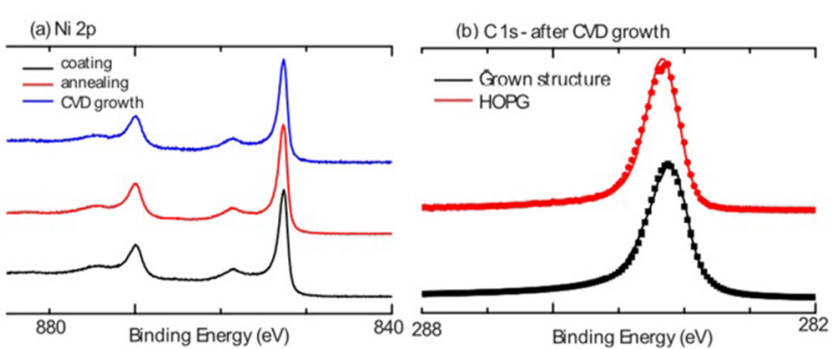

(c)

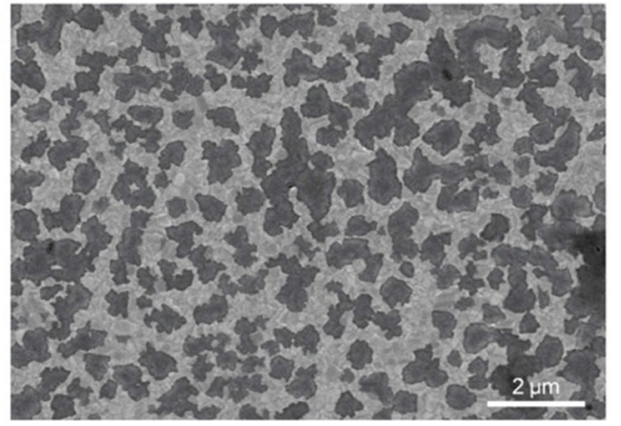

FIG. 1. (a) Ni 2p region of the XPS spectrum of as-deposited, annealed, and CVD coated Ni films. $\mathrm{Ni}$ is in metallic chemical state with two doublets originating from $\mathrm{c}^{-1} 3 \mathrm{~d}^{10} 4 \mathrm{~s}$ and $\mathrm{c}^{-1} 3 \mathrm{~d}^{9} 4 \mathrm{~s}^{2}$ final states. $2 \mathrm{p}_{3 / 2}$ at 852.6 and $858.6 \mathrm{eV}$, both with spin-orbit splitting of $+16.9 \mathrm{eV} .{ }^{17}$ (b) $\mathrm{C} 1 \mathrm{~s}$ region of the XPS spectrum after CVD growth shown together with a reference HOPG sample. Only the graphitic carbon peak at $284.2 \mathrm{eV}$ and the shake-up peak at $+6.5 \mathrm{eV}$ (not shown) are present. ${ }^{18}$ (c) SEM micrograph of the surface after CVD growth.

power, $360 \mathrm{~nm}$ spot size, and $3 \mathrm{~cm}^{-1}$ spectral resolution. $10 \times 10 \mu \mathrm{m}^{2}$ image was obtained by scanning the $\mathrm{x}-\mathrm{y}$ stage with $80 \times 80$ pixels. An integration time of $1 \mathrm{~s}$ per pixel was used. A typical Raman spectrum of graphene/graphite consists mainly of three peaks: ${ }^{19}$ (1) D peak around $1350 \mathrm{~cm}^{-1}$ due to breathing-like modes corresponding to transverse optical phonons near the $\mathrm{K}$ point of the Brillouin zone. It is a defect-activated intervalley double-resonance Raman process. (2) Its overtone, 2D peak around $2700 \mathrm{~cm}^{-1}$ (convolution of $2 \mathrm{D}_{1}$ and $2 \mathrm{D}_{2}$ peaks for graphite), arises from a high energy second-order process involving two inelastic scatterings, and it is also present when D peak is absent. Its position and shape depend on the number of graphene layers. (3) $\mathrm{G}$ peak around $1585 \mathrm{~cm}^{-1}$ represents the optical $\mathrm{E}_{2 \mathrm{~g}}$ phonons at the center of the Brillouin zone. Fig. 2(a) shows the Raman microspectroscopy of all these 3 peaks, which exhibits the material contrast shown in Fig. 1(c). A similar material contract can also be observed in the optical microscopy image in Fig. 2(b). Fig. 2(c) is an example of what an individual spectrum looks like on graphite/Ni and FLG/Ni zones. Similar to previous works on FLG on Ni films, we also observe the $2 \mathrm{D}$ band shifted to $2715 \mathrm{~cm}^{-1}$, a higher wavenumber with respect to graphene. ${ }^{20}$

KPFM measurements were performed with a customdesigned atomic force microscope operated at RT in ultra-high vacuum (UHV, $5 \times 10^{-10} \mathrm{mbar}$ ). To minimize the unintentional doping from air contaminants such as water, samples were annealed at $250^{\circ} \mathrm{C}$ for $1 \mathrm{~h}$ at UHV prior to KPFM measurements. ${ }^{21}$ Commercially available $\mathrm{Pt} / \mathrm{Ir}$ coated silicon cantilevers (Nanosensors PPP-NCLPt) were used as force sensors. The fundamental cantilever resonance frequency $\left(\mathrm{f}_{1}{ }^{\text {st }} \sim 160 \mathrm{kHz}\right)$ was excited to obtain topographical information, whereas the second flexural cantilever resonance $\left(\mathrm{f}_{2}{ }^{\text {nd }} \sim 990 \mathrm{kHz}\right)$ was used for the contact potential difference (CPD) detection in the non-contact regime. ${ }^{22,23}$ Nanonis Dual-OC4 was used as a digital phaselocked loop circuit for $\mathrm{f}_{1}{ }^{\text {st }}$ shift demodulation and as a lock-in amplifier for detecting the cantilever oscillation at $\mathrm{f}_{2}{ }^{\text {nd }}$. The amplitude of $\mathrm{f}_{2}^{\text {nd }}$ is minimized by adjusting the DC bias applied on the sample (i.e., brighter areas in CPD correspond to areas with higher WF), which is equal to the CPD between the tip and the sample.

Figs. 3(a) and 3(b) show the surface topography and CPD of the sample, respectively. The CPD image has the same dappled structure as images obtained by SEM (Fig. 1(c)). The brighter CPD contrast regions can be correlated to the graphite regions. Under the assumption that the tip remains unchanged with a nominal $\mathrm{WF}$ of $4.28 \mathrm{eV}$ for $\mathrm{Pt} / \mathrm{Ir}$ coated cantilever with an absolute error of $70 \mathrm{meV},{ }^{24}$ graphite's WF can be estimated to be around $4.35 \mathrm{eV}$, which is similar to the values given in literature. ${ }^{3,25}$ However, as can

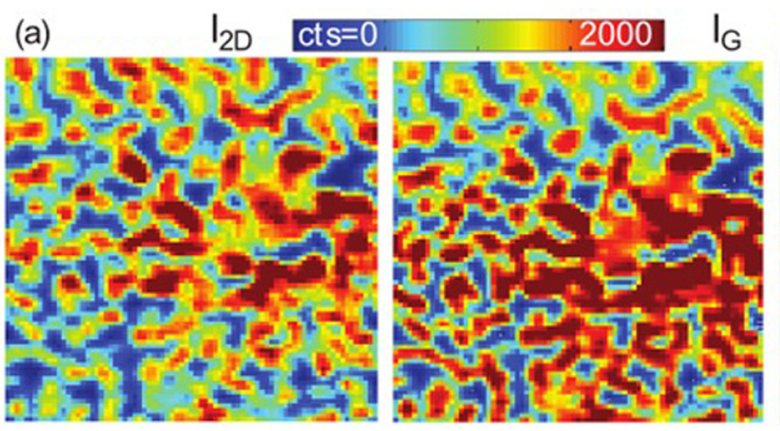

(b)

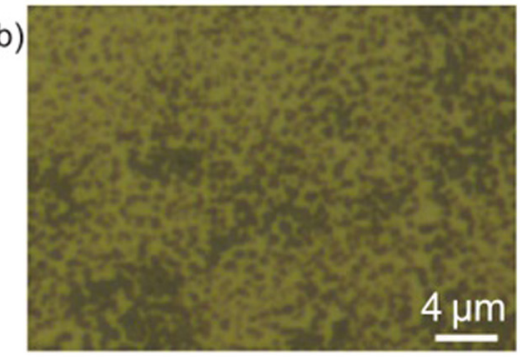

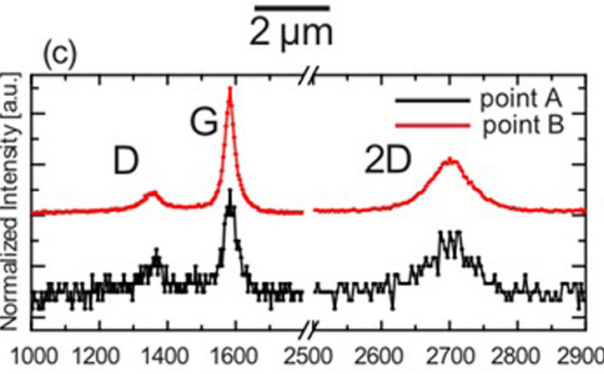

FIG. 2. Micrographs of the graphitic structures grown on Ni films with different techniques: (a) Raman spectroscopy images from typical Raman spectra presented in (c), and (b) optical microscopy. 

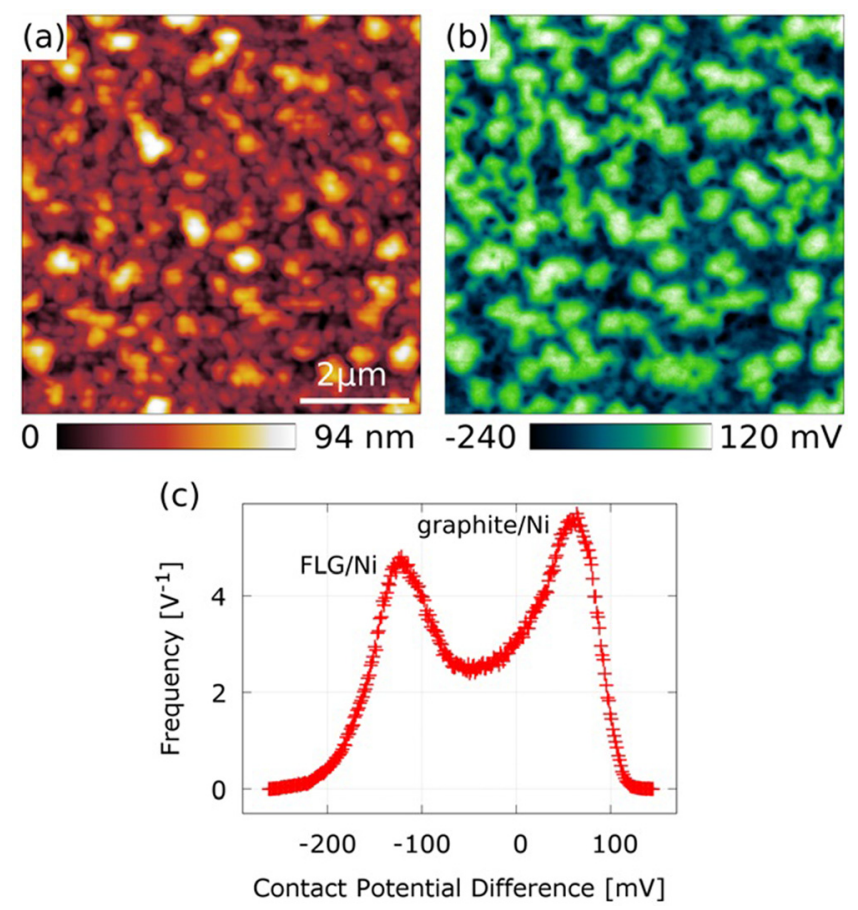

FIG. 3. KPFM images of Ni thin film covered with few layer graphene and graphite: (a) topography channel and (b) CPD channel. Higher CPD zones are graphite which mostly (but not always) appears as protruding out of the surface. (c) The histogram of the CPD channel.

also be seen in the CPD image, the WF is not homogeneous in the graphite areas and varies locally, therefore giving absolute values with error bars for such systems is questionable. This is especially true for averaged values acquired by photoelectron based techniques on such systems. The histogram of the CPD image in Fig. 3(c) clearly shows two peaks which can be correlated with the two distinct areas. The width $(\sim 50 \mathrm{meV})$ of the two peaks gives an estimation of the WF variations, which have to be added to the absolute error made by the calibration of the $\mathrm{Pt} / \mathrm{Ir}$ tip. Since undoped graphene and the metal substrate have different WF's, electrons will be transferred from one to another to equilibrate the Fermi levels as soon as graphene is physisorbed on a metal surface. This interaction will change the surface dipole moment of the metal surface, denoted by a potential step, $\Delta \mathrm{V}$. The work function measured from the vacuum will be equal to $\mathrm{WF}_{\text {metal }} \pm \Delta \mathrm{V}$, which is also equal to $\mathrm{WF}_{\text {graphene }} \pm \Delta \mathrm{E}_{\mathrm{f}}$. Here, $\Delta \mathrm{E}_{\mathrm{f}}$ denotes the change in graphene's WF upon adsorption on the surface, negative meaning electrons are donated by the metal substrate to graphene which becomes n-type. This is, however, a naive picture for graphene on $\mathrm{Ni}$, where the interface distances are small. At such small equilibrium separations, an overlap of the wave functions of the metal d-electrons and graphene $\pi$-electrons, i.e., orbital hybridization, occurs. Hybridized states exist in a certain energy range of the metal d-states and they push $\pi^{*}$ states to higher energies through repulsive energy level interactions, opening a $\pi$-gap with randomly distributed gap states. ${ }^{4}$ Such strong interactions (chemisorption) were claimed to reduce the work function of $\mathrm{Ni}$ from $5.5 \mathrm{eV}$ to $3.9 \mathrm{eV},{ }^{3,25}$ which is significantly lower than graphite. Since a Dirac point exists starting from the second graphene layer, ${ }^{7}$ $\Delta \mathrm{E}_{\mathrm{f}}$ can be defined as the difference between the Fermi level and the Dirac point; ${ }^{7}$ thus the following relation holds from the second layer upwards: Dirac point $>$ Fermi level $>$ vacuum level. The WF of graphite will eventually be reached as the number of graphene layers increases. ${ }^{26}$

In summary, the CPD contrast in Fig. 3(b) is a direct measurement of WF difference between graphite and FLG adsorbed on Ni. This leads to the conclusion that the WF of FLG/Ni is around 4.15 with a variation of $50 \mathrm{meV}$. KPFM is a very powerful technique for local WF estimations. In this letter, we used this technique to provide a strong firsthand evidence that the WF of FLG/Ni is lower than the WF of $\mathrm{Ni}$, of graphite and even of free standing graphene. Such peculiar property could be harvested in applications that demand low WF's such as thermionic emitters or transistor contacts for n-type semiconductors.

The authors would like to thank the Swiss Federal Office of Energy and the Federal Office for Education and Science for their financial support. This work was also supported by the Swiss National Foundation (SNF).

${ }^{1}$ J. Wintterlin and M.-L. Bocquet, Surf. Sci. 603, 1841-1852 (2009).

${ }^{2}$ N. C. Bartelt and K. F. McCarty, MRS Bull. 37, 1158-1165 (2012).

${ }^{3}$ G. Giovannetti, P. A. Khomyakov, G. Brocks, V. M. Karpan, J. van den Brink, and P. J. Kelly, Phys. Rev. Lett. 101, 026803 (2008).

${ }^{4}$ C. Gong, G. Lee, B. Shan, E. M. Vogel, R. M. Wallace, and K. Cho, J. Appl. Phys. 108, 123711 (2010).

${ }^{5}$ J. Rusz, A. B. Preobrajenski, M. L. Ng, N. A. Vinogradov, N. Mårtensson,

O. Wessely, B. Sanyal, and O. Eriksson, Phys. Rev. B 81, 073402 (2010).

${ }^{6}$ L. Zhanga, N. Vinogradov, A. Preobrajenski, S. Butorin, J. Zhu, and J.

Guo, Chem. Phys. Lett. 580, 43-47 (2013).

${ }^{7}$ J. Sun, J. B. Hannon, R. M. Tromp, P. Johari, A. A. Bol, V. B. Shenoy, and K. Pohl, ACS Nano 4, 7073-7077 (2010).

${ }^{8}$ L. Baraton, Z. B. He, C. S. Lee, C. S. Cojocaru, M. Châtelet, J.-L. Maurice, Y. H. Lee, and D. Pribat, Europhys. Lett. 96, 46003 (2011).

${ }^{9}$ J. J. Lander, H. E. Kern, and A. L. Beach, J. Appl. Phys. 23, 1305 (1952).

${ }^{10}$ K. Natesan and T. F. Kassner, Metall. Trans. 4, 2557-2566 (1973).

${ }^{11}$ A. Kirchner and B. Kieback, J. Nanomater. 2012, 953828.

${ }^{12}$ R. S. Weatherup, B. C. Bayer, R. Blume, C. Baehtz, P. R. Kidambi, M. Fouquet, Ch. T. Wirth, R. Schlögl, and S. Hofmann, ChemPhysChem 13, 2544-2549 (2012).

${ }^{13}$ B. Eren, L. Marot, R. Steiner, T. de los Arcos, M. Düggelin, D. Mathys, K. N. Goldiee, V. Olivieri, and E. Meyer, Chem. Phys. Lett. 609, 82-87 (2014).

${ }^{14}$ H. J. Park, J. Meyer, S. Roth, and V. Skákalová, Carbon 48, 1088-1094 (2010).

${ }^{15}$ H. Seiler, J. Appl. Phys. 54, R1-R18 (1983).

${ }^{16}$ P. J. Cumpson, Surf. Interface Anal. 29, 403-406 (2000).

${ }^{17}$ S. Hüfner, Photoelectron Spectroscopy Principles and Applications (Springer Verlag, Heidelberg, 1996).

${ }^{18}$ B. Eren, D. Hug, L. Marot, R. Pawlak, M. Kisiel, R. Steiner, D. M. Zumbühl, and E. Meyer, Beilstein J. Nanotechnol. 3, 852-859 (2012).

${ }^{19}$ A. C. Ferrari, J. C. Meyer, V. Scardaci, C. Casiraghi, M. Lazzeri, and F. Mauri, Phys. Rev. Lett. 97, 187401 (2006).

${ }^{20}$ L. Baraton, Z. He, C. S. Lee, J.-L. Maurice, C. S. Cojocaru, A.-F. Gourgues-Lorenzon, Y. H. Lee, and D. Pribat, Nanotechnology 22, 085601 (2011).

${ }^{21}$ D. Martínez-Martína, R. Longuinhos, J. G. Izquierdo, A. Marele, S. S. Alexandre, M. Jaafar, J. M. Gómez-Rodríguez, L. Bañares, J. M. Soler, and J. Gomez-Herrero, Carbon 61, 33-39 (2013).

${ }^{22}$ A. Kikukawa, A. Hosaka, and R. Imura, Rev. Sci. Instrum. 67, 1463 (1996).

${ }^{23}$ Ch. Sommerhalter, Th. W. Matthes, Th. Glatzel, A. Jäger-Waldau, and M. Ch. Lux-Steiner, Appl. Phys. Lett. 75, 286 (1999).

${ }^{24}$ S. Sadewasser, Th. Glatzel, M. Rusu, A. Jäger-Waldau, and M. Ch. LuxSteiner, Appl. Phys. Lett. 80, 2979-2981 (2002).

${ }^{25}$ C. Oshima and A. Nagashima, J. Phys.: Condens. Matter 9, 1-20 (1997).

${ }^{26}$ H. Hibino, H. Kageshima, M. Kotsugi, F. Maeda, F.-Z. Guo, and Y. Watanabe, Phys. Rev. B 79, 125437 (2009). 